\title{
MS38-04 | Eiger2 CdTe Detectors: Tools for HARd X-RAy Studies
}

Brandstetter, Stefan (DECTRIS Ltd, Baden-Daettwil, CH); Wagner, Lucas (DECTRIS Ltd, Baden-Dättwil, CH); Kaspar, Sebastian (DECTRIS Ltd, Baden-Dättwil, CH)

Hybrid Photon Counting (HPC) X-ray detectors have transformed synchrotron research in the last decade. They provide noise-free detection and permit new data acquisition modes such as continuous data collection and extremely high frame rates. The new HPC detector family EIGER2 enables even more ambitious X-ray science with different sensor materials suited for high-energy applications. These detectors combine the advantages of previous generations of detectors. They offer pixels sizes of $75 \mu \mathrm{m} \times 75 \mu \mathrm{m}$, frame rates in the kilohertz range, negligible dead time (100 ns), and count rates of up to $10^{7}$ photons per pixel and second through DECTRIS retrigger technology. Moreover, EIGER2 technology is compatible with silicon and cadmium telluride (CdTe) sensor material, which offers high quantum efficiency up to around $80 \mathrm{keV}$. Two adjustable energy thresholds extend the usability of the EIGER2 detector family, allowing reduction of the cosmic background and higher harmonics. These properties not only empower new fields of X-ray photon research like X-ray diffraction computed tomography (XRD-CT) but will also advance established methods like X-ray crystallography in the high-energy range.

Here, we present results from first experiments and characterization methods. Detector properties like count rate capability, readout, and spatial resolution are demonstrated with dedicated key experiments. With an EIGER2 X $1 \mathrm{M}$ CdTe we collected excellent high-pressure data from crystals in a diamond anvil cell. Combined with characterization measurements at beamlines and in the laboratory, these results show how the latest HPC CdTe detectors empower advances in X-ray micro- and nanoanalysis. 